\title{
Efficient Face Recognition System using Artificial Neural Network
}

\author{
S.Adebayo Daramola \\ Department of Electrical and \\ Information Engineering, \\ Covenant University Ota, \\ Nigeria
}

\author{
O. Sandra Odeghe \\ Department of Electrical and \\ Information Engineering, \\ Covenant University Ota, \\ Nigeria
}

\begin{abstract}
Effective facial feature is needed to build a robust face recognition system capable of suppress the effect of illumination and pose variation. In this paper, a robust face recognition system is proposed. In the proposed system, two level haar wavelet transform is used to decompose frontal face image into seven sub-image bands. Thereafter eigenface feature is extracted from these bands. The feature is used as input to the classification algorithm based on Back Propagation Neural Network (BPNN). The proposed system has been tested using 150 frontal face samples with illumination and pose variation. The results obtained are very encouraging.
\end{abstract}

\section{General Terms}

Recognition, Security.

\section{Keywords}

Eigenface, Haar Wavelet Transform and Neural Network.

\section{INTRODUCTION}

Face image is a biometrics physical feature use to identify people. Major components present in face image space include eyes, nose and mouth. Among biometrics trait face is the most natural means of identify people. Face identification can be done automatically using digital camera as the capturing device. The processed face image feature is used for final matching. High intra-class variation within face images of the same person is the major problem in this research area. Pose and illumination are responsible for high intra-class variation in facial images [1]

Automatic face recognition can be done using different combination of feature extraction methods and classifiers. In many cases features are extracted from whole facial space using Principal Component Analysis (PCA) [2][3]. On other hand it involves extraction of features from face components [4][5]. Matching of feature with subject template may be on a pixel to pixel basis using Euclidean distance measure [6]. Prominent classifiers like Support Vector Machine (SVM), Artificial Neutral Network (ANN) are used for face matching [7][8].

In [9], face recognition method is proposed. Legendre, Zernike and pseudo-Zernike moments features were extracted. They use discrete wavelet transform (DWT) to obtain low level image resolution before extract moments features from face image while in [10] they presented an enhanced fused approach for face recognition based on voronoi diagram and wavelet moment invariants. Discrete wavelet transform and moment invariants are used for feature extraction of the facial face. Gabor filter, log Gabor filter and discrete wavelet transform are applied to face image prior to dimensionality reduction by Principal Component Analysis (PCA) in the paper presented in [11]. The approximation coefficients in discrete wavelet transform is extracted and it was used to compute the face recognition accuracy instead of using all the coefficients. In [12] they proposed Face Recognition Algorithms that uses combination of Gabor and eigenfaces to obtain the feature vector. This feature vector is feed to a multilayer perceptron to carry out the face recognition or identity verification tasks. The proposed system is meant to provide robustness against changes in illumination and facial expressions. The paper presented in [13] is used tests the performance of Principle Component Analysis (PCA) based technique for face recognition. Recognition of human faces with two facial expressions is done. Classification of face images are carried out using distance based classifier and Neural Network (NN).

In this work, better method for face recognition is proposed using our database. Eigenface feature is extracted from subband images of the second level Haar wavelet transform decomposition product. Neural Network is used as the classification algorithm. The feature is robust enough to suppress the effect of varying pose and illumination. The rest of the paper is organized as follows: Section 2 describes the system component. Face image decomposition is presented in section 3, section 4 introduces the feature extraction method, and section 5 describes classification process based on Neural Network. The experimental results are shown in section 6 . Finally, conclusion is presented in section 7 .

\section{SYSTEM DESCRIPTION}

The proposed face recognition system flow diagram is shown in fig.1. It consists of frontal face image acquisition process, feature extraction algorithm and classification algorithm based on Back Propagation Neural Network (BPNN). 


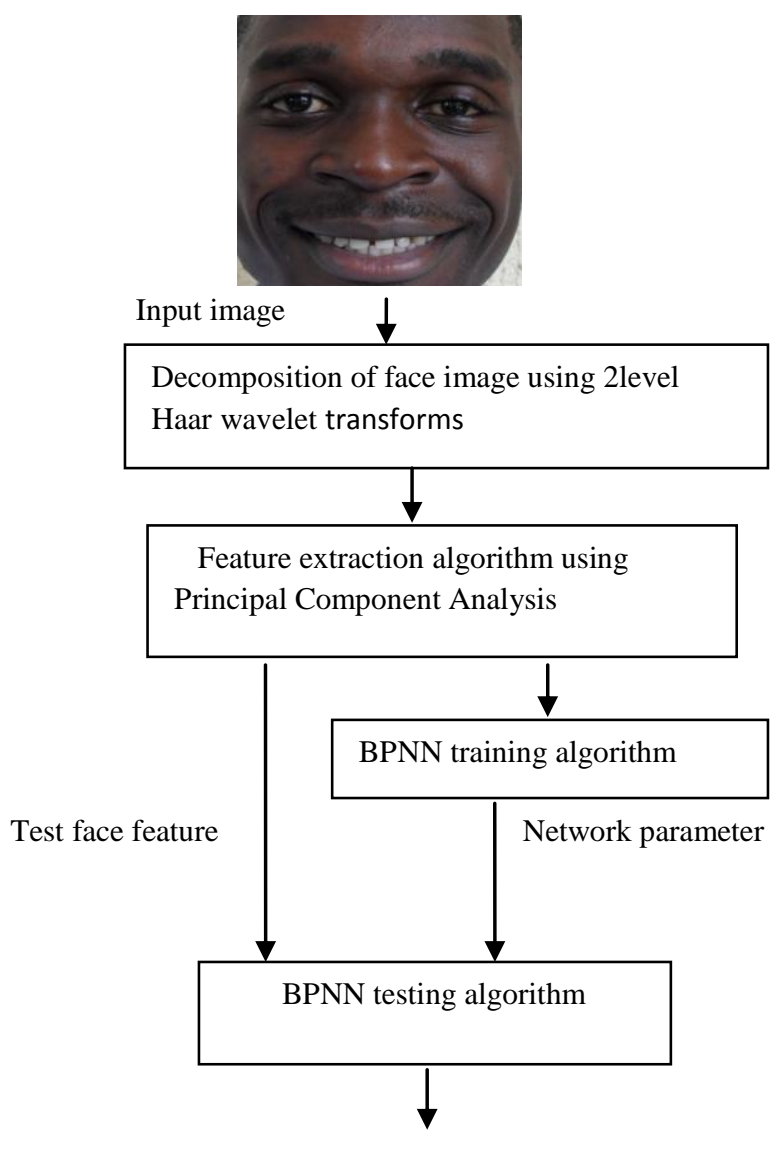

Output class

Fig.1: Flow diagram of the proposed face recognition system.

\section{FACE IMAGE DECOMPOSITION}

Wavelet transform is used to decompose original grayscale image into four regions namely vertical high frequencies, horizontal high frequencies, diagonal high frequencies and approximate region. The approximation region contains the lowest frequencies feature. At the second level decomposition, the approximation region at the upper left corner of the image is decomposed into four smaller regions. Therefore at second decomposition there are seven sub-image bands. Also the size of the original image is still equal to the sum of sizes of all sub-image bands. Further decomposition or better technique can be used to extract prominent features from the sub-image band [13][14].

In this work two levels haar wavelet transform is used to decompose original frontal face image into seven sub-bands. One example is shown in fig. 2 .

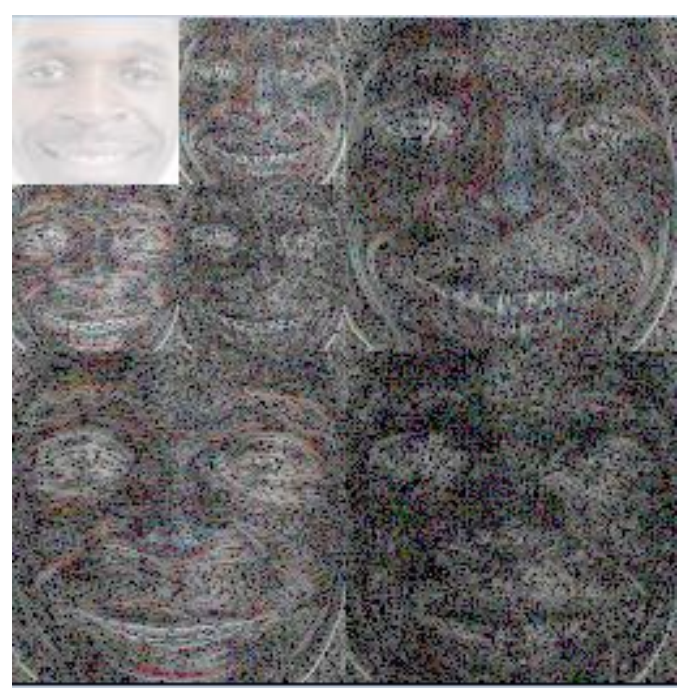

Fig.2: Facial image decomposition

\section{FEATURE EXTRACTION PROCESS.}

Principal Component Analysis (PCA) is used in this work as the feature extractor. PCA is performed on the output of face image decomposition process to produce eigenvector. It is possible in PCA to recover original image using dominant eigenvector. Therefore eigenvector is a good representation of the original image. Eigenvector is chosen based on the eigenvalues. The eigenvalues contain the variation in the original data set. Given a set of $\mathrm{N}$ dimensional image bands $\mathrm{X}_{\mathrm{i}}$. The covariance matrix $(\mathrm{C})$ is obtained using mean vector $(\mu)$ of the image bands $X_{i}$ as in (1).

$$
C=\frac{1}{N} \sum_{i}^{N}\left(X_{i}-\mu\right)\left(X_{i}-\mu\right)^{T}
$$

The eigenvalues and the eigenvector of the covariance matrix (C) are estimated by sorting eigenvalues and corresponding eigenvectors in descending order.

We find the eigenvector of the sub-image bands based on the size using the following steps. Fig.3 shown the sub-image bands representation.

\begin{tabular}{|l|l|l|}
\hline $\mathrm{H}_{1}$ & $\mathrm{H}_{3}$ & \multirow{2}{*}{$\mathrm{V}_{5}$} \\
\cline { 1 - 2 } $\mathrm{H}_{2}$ & $\mathrm{H}_{4}$ & \\
\hline $\mathrm{V}_{6}$ & & $\mathrm{~V}_{7}$ \\
\hline
\end{tabular}

Fig 3: Representation of sub-image bands 
(i) Convert each of the sub-band image matrix to image vector to obtain (2) and (3).

$$
\begin{aligned}
& \mathrm{H}_{1}=\mathrm{h}_{11}, \mathrm{~h}_{12}, \mathrm{~h}_{13} \ldots \ldots \ldots \ldots \ldots \ldots \mathrm{h}_{1 \mathrm{~m}} \\
& \mathrm{H}_{2}=\mathrm{h}_{21}, \mathrm{~h}_{22}, \mathrm{~h}_{23} \ldots \ldots \ldots \ldots \ldots \ldots . . . \mathrm{h}_{2 \mathrm{~m}}
\end{aligned}
$$

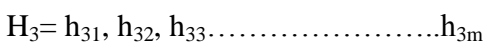

$$
\begin{aligned}
& \mathrm{H}_{4}=\mathrm{h}_{41}, \mathrm{~h}_{42}, \mathrm{~h}_{43} \ldots \ldots \ldots \ldots \ldots \ldots . . . . . . . . . . \\
& \mathrm{V}_{5}=\mathrm{v}_{51}, \mathrm{v}_{52}, \mathrm{v}_{53} \ldots \ldots \ldots \ldots \ldots \ldots \ldots \mathrm{v}_{5 \mathrm{n}} \\
& \mathrm{V}_{6}=\mathrm{v}_{61}, \mathrm{v}_{62}, \mathrm{v}_{63} \ldots \ldots \ldots \ldots \ldots \ldots \mathrm{v}_{6 \mathrm{n}} \\
& \mathrm{V}_{7}=\mathrm{v}_{71}, \mathrm{v}_{72}, \mathrm{f}_{73} \ldots \ldots \ldots \ldots \ldots \ldots \ldots \mathrm{v}_{7 \mathrm{n}}
\end{aligned}
$$

(ii) Obtain the eigenvector $\left(\mathrm{EH}_{1} \ldots . . \mathrm{EH}_{\mathrm{n}}\right)$ from the covariance matrix of sub- image bands $\mathrm{H}_{1}, \mathrm{H}_{2}, \mathrm{H}_{3}$ and $\mathrm{H}_{4}$.

(iii) Obtain the eigenvector $\left(\mathrm{EV}_{1} \ldots \ldots \mathrm{EV}_{\mathrm{k}}\right)$ from the covariance matrix of sub- image bands $\mathrm{V}_{5}, \mathrm{~V}_{6}$ and $\mathrm{V}_{7}$.

(iv) The extracted feature vector is formed by concatenation of the eigenvectors as given in (4).

$\mathrm{F}=\left[\mathrm{EH}_{1} \ldots \ldots \mathrm{EH}_{\mathrm{n}}, \mathrm{EV}_{1} \ldots \ldots . . \mathrm{EV} \mathrm{k}_{\mathrm{k}}\right]$

The robust feature extraction method presented here is different from existing methods [9][13].

\section{BACK-PROPAGATION NEURAL NETWORK FOR FACE RECOGNITION}

Back Propagation Neural Network (BPNN) is a multilayered, feed forward Neural Network (NN). BPNN consists input layer, one or more hidden layer followed by output layer. The layers contain identical computing nodes (neurons) connected in such way that output neuron in one layer send signal to input of every neuron in the next layer. The input layer of the network serves as the signal receptor while the output layer passes out the result from the network [16].

In this work, we used three layers BPNN as the classifier, the number of node in the input layer is equal to dimension of the feature vector that characterizes the face image space. The

Table.1 Experimental results.

\begin{tabular}{|c|c|c|c|}
\hline $\begin{array}{c}\text { Number } \\
\text { of face } \\
\text { images }\end{array}$ & $\begin{array}{c}\text { Face } \\
\text { matched } \\
\text { correctly }\end{array}$ & $\begin{array}{c}\text { Face mis- } \\
\text { matched }\end{array}$ & $\begin{array}{c}\text { Recognition } \\
\text { rate(\%) }\end{array}$ \\
\hline 30 & 25 & 5 & 83.3 \\
\hline 60 & 52 & 8 & 86.7 \\
\hline 90 & 80 & 10 & 88.9 \\
\hline 120 & 106 & 14 & 88.3 \\
\hline 150 & 126 & 24 & 84.0 \\
\hline
\end{tabular}

number of the node in the hidden layer is set by trial and error during training. The number of node in the out layer is equal to the number of the subjects in the database. During training process, The BPNN learning algorithm adjusts weight and bias of each of the neurons in order to minimize the Mean Square Error (MSE) between the targets and predicts output. In the recognition phase, the features from the query face image that is to be tested is fed into the network without having any target output, BPNN testing algorithm found the closest matching output using the weights and thresholds that have stored and provided the corresponding recognized face.

\section{EXPERIMENTAL RESULTS}

Experiments are carried out for the training and testing of frontal face images. We analyzed the performance of the proposed algorithm using our database. The data (faces) in the database used for training and testing are the face images of students of Covenant University, department of Electrical and Information Engineering taken in a controlled environment of different illuminations. Fifty (50) individual faces were taken with a digital camera. Each individual has seven (7) images with different face expressions and lightings as shown in fig.4.
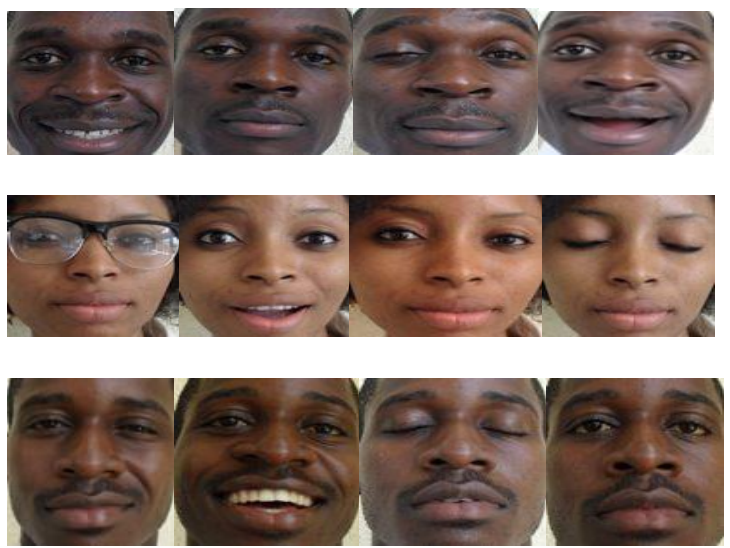

Fig.4: Sample of face images from our database.

experıment is as snown in 1 abie 1 .

\section{CONCLUSION}

Efficient face recognition system is needed in order to increase security in access control and financial transactions. The proposed system is robust enough to improve the current situation. Face recognition using Artificial Neural Network and robust feature vector has been developed to overcome limitations due pose variation. Encouraging results have been obtained by using four images for training and three images for testing purposes per class 


\section{REFERENCES}

[1] A.M Patil, S.R Kolhe and P.M Patil, "2D Face Recognition Techniques: A Survey", International Journal of Machine Intelligence, Volume 2, Issue 1, 2010, pp-74-83.

[2] M. Agarwal, N. Jain, M. Kumar and H. Agrawal, "Face Recognition Using Eigen Faces and Artificial Neural Network", International Journal of Computer Theory and Engineering, Vol. 2, No. 4, August, 2010, pp624629.

[3] P.Latha, Dr..L.Ganesan and Dr..S. Annadurai, "Face Recognition using Neural Networks", Signal Processing: An International Journal (SPIJ) Volume (3): Issue (5) pp153-160.

[4] S. Kumar Paul , M. Shorif Uddin and S. Bouakaz, "Extraction of Facial Feature Points Using Cumulative Histogram", IJCSI International Journal of Computer Science Issues, Vol. 9, Issue 1, No 3, January 2012 pp4451.

[5] R.1 Dewi Agushinta, Adang Suhendra, Sarifuddin Madenda, H.S Suryadi, "Face Component Extraction Using Segmentation Method on Face Recognition System", Journal of Emerging Trends in Computing and Information Sciences, Volume 2 No. 2, pp67-72.

[6] Dr. H. B. Kekre1, S. D. Thepade, T. Chopra, "Face and Gender Recognition Using Principal Component Analysis", International Journal on Computer Science and Engineering, Vol. 02, No. 04, 2010, pp959-964.

[7] K. Ramirez-Gutierrez, D. Cruz-Perez, J. OlivaresMercado, M. Nakano-Miyatake, and H. Perez-Meana, "A Face Recognition Algorithm using Eigenphases and Histogram Equalization", International Journal of Computers Issue 1, Volume 5, 2011 pp34-41.

[8] M. A. Kashem, M. N. Akhter, S. Ahmed, and M. M. Alam, "Face Recognition System Based on Principal
Component Analysis (PCA) with Back Propagation Neural Networks (BPNN)", Canadian Journal on Image Processing and Computer Vision, Vol.2, No.4, 2011.

[9] A. Bastani and E. F. Behbahani, "An Efficient Feature Extraction Method with Orthogonal Moments and Wavelet Transform for Human Face Recognition System", European Journal of Scientific Research, Vol.52 No.3 (2011), pp.313-320.

[10] K. Meethongjan, M. Dzulkifli, A. Rehman and T. Saba, "Face Recognition based on Fusion of Voronoi Diagram Automatic Facial and Wavelet Moment Invariants", International Journal of Video \& Image Processing and Network Security IJVIPNS (10) 4, 2010.

[11] D. Murugan, Dr. S Arumugam, K. Rajalakshmi and T.I.Manish, "Performance evaluation of face recognition using Gabor filter, log Gabor filter and discrete wavelet transform”, International Journal of Computer Science and Information (IJSCI) (2)1, 2010.

[12] G. Aguilar-Torres, K. Toscano-Medina, G. SanchezPerez, M. Nakano-Miyatake, and H. Perez-Meana, "Eigenface-Gabor Algorithm for Features Extraction in Face Recognition", International Journal of Computers, Issue 1, Volume 3, 2009.

[13] R. M Rahman, Anirban Das M Russel and Md. Shazzad Maruf, "Face Recognition for Single and Different Facial Expressions", Global Journal of Computer Science and Technology, Vol. 10 Issue 9, 2010, pp16-21.

[14] S. E Umbaugh "Computer Imaging: Digital Image Analysis processing", A CRC press book.

[15] Raju, U.S.N., A. Srikrishna, V. Vijaya Kumar and A. Suresh, "Extraction of Skeleton Primitives on Wavelets", Journal of Theoretical and Applied Information Technology, pp1065 - 1074, 2008.

[16] R. C. Gonzalez and R. E. Woods, "Digital Image processing”, $3^{\text {rd }}$ Edition, Pearson International Edition. 